\title{
Erratum
}

\section{Vibrio parahaemolyticus cqsA controls production of quorum sensing signal molecule 3-hydroxyundecan-4-one and regulates colony morphology}

\author{
Kui Wu ${ }^{1,2,4_{\star}}$, Yangyun Zheng ${ }^{1}$, Qingping $\mathrm{Wu}^{2}{ }^{2}$, Haiying $\mathrm{Chen}^{1}$, Songzhe $\mathrm{Fu}^{3}$, Biao Kan ${ }^{4}$, \\ Yongyan Long ${ }^{1,4}$, Xiansheng $\mathrm{Ni}^{1}$, and Junling $\mathrm{Tu}^{1}$ \\ ${ }^{1}$ The Collaboration Unit for Field Epidemiology of State Key Laboratory for Infectious Disease Prevention and Control, \\ Nanchang Center for Disease Control and Prevention, Nanchang, 330038, P. R. China \\ ${ }^{2}$ State Key Laboratory of Applied Microbiology South China, Guangdong Institute of Microbiology, Guangzhou, 510070, P. R. China \\ ${ }^{3}$ College of Marine Technology and Environment, Dalian Ocean University, Dalian, 116023, P. R. China \\ ${ }^{4}$ State Key Laboratory for Infectious Disease Prevention and Control, National Institute for Communicable Disease Control and Prevention, \\ Chinese Center for Disease Control and Prevention, Beijing, 102206, P. R. China
}

In the article by Wu et al. published in Journal of Microbiology 2019; $57,1105-1114$, the figure 8 is unfortunately incorrect. The figure 8 should be corrected as below.

We apologize for any inconvenience that this may have caused.

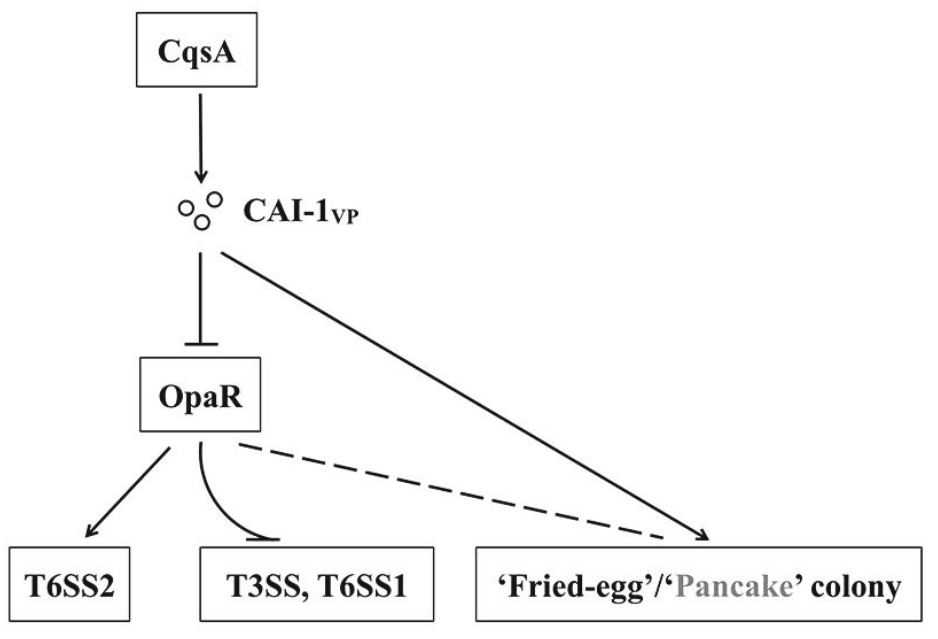

